\title{
Adolescent with Tourette Syndrome and Bipolar Disorder: A Case Report
}

\author{
Se-Hoon Shim, Young-Joon Kwon \\ Department of Psychiatry, Soonchunhyang University Cheonan Hospital, College of Medicine, Soonchunhyang University, Cheonan, Korea
}

\begin{abstract}
Tourette syndrome consists of multiple motor tics and one or more vocal tics. Psychopathology occurs in approximately $90 \%$ of Tourette syndrome patients, with attention-deficit/hyperactivity, mood, and obsessive-compulsive disorders being common. Additionally, Tourette syndrome and bipolar disorder may be related in some individuals. However, it is unclear why bipolar disorder may be overrepresented in Tourette syndrome patients, and more research is needed. Herein, we report the case of a 15-year-old boy diagnosed with both Tourette syndrome and bipolar disorder, whose symptoms improved with aripiprazole, atomoxetine, and valproate. The patient was diagnosed with Tourette syndrome at 8 years of age when he developed tics and experienced his first depressive episode. The patient had a poor response to a variety of antidepressants and anti-tic medications. A combination of valproate and aripiprazole stabilized both the patient's tics and mood symptoms. It is important to assess individuals with Tourette syndrome for other disorders, including bipolar disorder. The treatment of children and adolescents with both Tourette syndrome and bipolar disorder is an important clinical issue.
\end{abstract}

KEY WORDS: Bipolar disorder; Child; Adolescent; Tourette syndrome.

\section{INTRODUCTION}

Tourette syndrome is a childhood-onset neuropsychiatric disorder first reported by the French physician Georges Gilles de la Tourette in 1885. It is characterized by a combination of multiple motor and one or more vocal tics. Tourette syndrome is distinguished by erratic movements of the whole body accompanied by sporadic instances of aimless spitting and shouting and is unpleasant to those around the patient. Moreover, it is a chronic disorder with repetitive cycles of waxing and waning distress for both the patient and the patient's family. The onset of Tourette syndrome is during childhood, and the prevalence is 3-fold higher in boys $(4-5$ per 10,000) than in girls, ${ }^{1)}$ although a statistically assured epidemiologic study has not been conducted in South Korea.

Bipolar disorder is difficult to diagnose in children and adolescents due to mood swings that are incompatible with the Diagnostic and Statistical Manual of Mental Disorders 4th edition (DSM-IV) diagnostic criteria for bi-

\footnotetext{
Received: February 24, 2014 / Revised: May 19, 2014

Accepted: May 26, 2014

Address for correspondence: Se-Hoon Shim, MD, PhD Department of Psychiatry, Soonchunhyang University Cheonan Hospital, 31 Suncheonhyang 6-gil, Dongnam-gu, Cheonan 330-903, Korea

Tel: +82-41-570-2280, Fax: +82-41-570-3878

E-mail:shshim2k@naver.com
}

polar disorder, difficulty differentiating bipolar symptoms from normal developmental changes, and the inability of children and adolescents to adequately describe their symptoms. Recently, pediatric bipolar disorder has been linked to suicide and increased risk of psychotic symptoms. To date, no systematic research on the prevalence of pediatric bipolar disorder in South Korea has been conducted. However, according to studies outside of Korea, pediatric bipolar disorder is significantly increasing, with a predicted prevalence of approximately $1 \%$, similar to adults, although these results are not without controversy. ${ }^{2,3)}$

Patients with Tourette syndrome commonly have additional neuropsychiatric disorders; a global study involving 3,500 Tourette syndrome patients demonstrated a comorbidity rate of $88 \%$. The most frequent comorbidity was attention deficit hyperactivity disorder (ADHD), and a variety of other neuropsychiatric disorders, such as obsessive-compulsive disorder and mood disorders, were observed with Tourette syndrome. Increased incidence of anxiety, insomnia, hostility, coprolalia, self-injurious behavior, and personality disorders has also been observed. ${ }^{4}$ Depressive and manic symptoms were especially common comorbidities, and it has been suggested that these symptoms are inevitable rather than being a result of motor or vocal tics. ${ }^{5}$ When Tourette syndrome is accompanied by bipolar disorder, stress induced by manic symp-

(a) This is an Open-Access article distributed under the terms of the Creative Commons Attribution Non-Commercial License (http://creativecommons.org/licenses/by-nc/3.0) which permits unrestricted non-commercial use, distribution, and reproduction in any medium, provided the original work is properly cited. 
toms is thought to exacerbate tics, ${ }^{6)}$ and conversely, tics are thought to stimulate or protract manic symptoms by giving rise to serious problems in both social and educational situations. ${ }^{7)}$ Therefore, adequate treatment of each disorder is required to prevent a vicious cycle that will impede treatment of the individual disorders.

Although up-to-date treatments are available for Tourette syndrome, ${ }^{8)}$ few studies have investigated pediatric bipolar disorder, and there are no reports available on the treatment of children and adolescents with Tourette syndrome and manic symptoms. Therefore, we report the diagnosis and treatment of a 15 -year-old boy with Tourette syndrome and bipolar disorder.

\section{CASE}

A 15-year-old male presented with vocal tics and motor tics consisting of shouting, grimacing, and twisting the neck to the right. He was reluctant to go to school because he did not get along with friends and his violent behavior and irritable mood frequently interfered with his home life.

The patient had febrile convulsions at the age of 2 years and received treatment in a pediatric clinic. Although he had delayed language development, he did not receive any treatment.

His father had a history of alcohol abuse and severe mood swings. His parents separated when the patient was 5 years old, and they divorced 3 years later.

During his parents' separation, the patient began frequent blinking of his eyes, and his twin brother yawned frequently. While the tics of the patient's twin brother were transient and improved spontaneously over time, the patient's tics began to include loud sounds, and even coprolalia. The patient's father scolded him harshly and physically punished him in an attempt to correct his behavior. As a consequence, he was anxious and developed insomnia.

In kindergarten, the patient began to manifest inattention, hyperactivity, and impulsiveness. The patient was bullied by his classmates in school because of his tics and restless behavior during class. At that time, the patient was diagnosed with Tourette syndrome, ADHD, and anxiety disorder.

He underwent outpatient treatment with 20-mg imipramine, 15-mg aripiprazole, and 27-mg methylphenidate irregularly from 2006 to 2008 . Although the patient's motor and vocal tics improved with treatment, he remained depressed and refused to go to school because he believed that he was a slow learner, and his facial tics made him ugly. When he went back to school without medication, he often fought with his classmates due to his irritability. The patient took a leave of absence from middle school in 2008 when his vocal tics increased and became louder. The patient was prescribed 50-mg sertraline, 4.5-mg haloperidol, and 1-mg benztropine intermittently at a local mental health clinic. However, his tics waxed and waned, and he became increasingly irritable. The patient also screeched and was ill-tempered with his mother, resulting in a first hospitalization. The patient's electroencephalography and brain magnetic resonance imaging tests were normal, and clinical psychological tests revealed an intelligence quotient (IQ) of 89 (verbal IQ, 92 and performance IQ, 87) on the Korean Education Development Institute-Wechsler Intelligence Scale for Children and abnormal findings on the ADHD Diagnostic System, Stroop, and color-trail tests. The patient demonstrated impaired understanding of social circumstances and a pattern of actions associated with unstable, discordant, and impulsive behaviors. $\mathrm{He}$ felt vulnerable and was sensitive to the attention of others. He was easily stimulated to aggressive behavior and became agitated in response to neutral social stimulation. The patient was emotionally unstable, overly sensitive, and felt on edge. During this hospitalization, he was placed on 50-mg imipramine, 4-mg risperidone, and 27-mg methylphenidate and discharged following improved symptoms. When he returned to school, the patient was placed in a special class because of conflicts with, and bullying by, his classmates. The patient felt depressed and unmotivated, and his vocal and motor tics continued. The patient continued to receive medication at a local clinic and was prescribed 20-mg escitalopram, 6-mg risperidone, 10-mg diazepam, and 2-mg benztropine.

The patient graduated from middle school in 2012, discontinued his school work, and stayed home because his vocal tics increased, and he was extremely distracted. Now that the patient remained home, he had an increasing number of conflicts with his parents. When the patient became dysphoric, he easily lost his temper, and used rapid, pressured, and loud speech with his family, which interfered with family life. Moreover, as he grew increasingly talkative and uncontrollable, he fought with his twin brother and displayed unstable moods. He manifested impulsivity, aggressive behavior, distractibility, irritability, and abnormally increased energy. His motor and vocal tics increased in frequency, and he would shout "ahk" multiple times per day, shake his whole body, and display self-injurious behavior by striking walls with his elbow when he felt frustrated. Eventually, the patient became unmanage- 
able and was hospitalized for the second time.

The patient's diagnosis was changed to bipolar II disorder, and escitalopram and risperidone were discontinued gradually, while aripiprazole was added. The Yale Global Tic Severity Scale score (YGTSS) ${ }^{9)}$ was 17 for vocal tics and 15 for motor tics. The Young Mania Rating Scale score (YMRS) ${ }^{10)}$ was 22.

The motor tics, which were disturbing to others, and the shouting vocal tics decreased by one-third while taking 20-mg aripiprazole and 20-mg atomoxetine; additionally, an improvement in manic behavior was noted.

When the aripiprazole and atomoxetine doses were increased to 25 and $40 \mathrm{mg}$, the tics and manic symptoms became aggravated. Therefore, the doses of aripiprazole and atomoxetine were decreased to 20 and $25 \mathrm{mg}$, respectively, to which $250 \mathrm{mg}$ of valproate was added.

The patient was stabilized on 500-mg valproate, $20-\mathrm{mg}$ aripiprazole, and 25-mg atomoxetine. The patient was discharged when the YGTSS ${ }^{9}$ decreased to 5 for vocal tics and 3 for motor tics and the YMRS ${ }^{10)}$ decreased to 9. At present, the patient is receiving outpatient treatment and rehabilitation but does not attend school.

\section{DISCUSSION}

This case involves a child initially diagnosed with a tic disorder who later also received a diagnosis of bipolar disorder. The patient's initial diagnosis was Tourette syndrome with ADHD accompanied by depression and anxiety. The administration of antidepressants revealed the manic symptoms of bipolar disorder including hyperactivity, excitation, distractibility, decreased need for sleep, and pressured speech. Ultimately, the patient's symptoms improved following the administration of an antipsychotic agent reported to be effective in tic and bipolar disorders.

Although a clear relationship between Tourette syndrome and bipolar disorder has not been defined, mood swings and aggressiveness frequently occur in patients with Tourette syndrome, and many studies suggest that the two disorders are related. ${ }^{4,7,8)}$ Comings and Comings ${ }^{5)}$ reported that $19.1 \%$ of patients with Tourette syndrome experienced manic symptoms, whereas the control group displayed no signs of manic symptoms; they suggested that Tourette syndrome and bipolar disorder may have a similar gene locus. The 16q22-23 region has been proposed as a candidate region for the primary or modifying genetic factors resulting in Tourette syndrome and bipolar disorder phenotypes. ${ }^{11)}$ Although the susceptibility se- quence of Tourette syndrome has not been identified by gross chromosomal rearrangement studies, chromosomal rearrangements related to Tourette syndrome, including cytogenetically invisible deletions, duplications, and inversions, appear to be associated with bipolar disorder. ${ }^{12)}$

A study of 205 patients with Tourette syndrome identified $15(7 \%)$ patients with bipolar disorder, a four-fold higher prevalence than in the normal population, and males with Tourette syndrome were at a greater risk for bipolar disorder than were females. These results indicate a connection between the two diseases. $7,8,11,13-15)$

Another study found that one (3\%) of 38 control group patients had bipolar disorder, and four (13\%) of 32 childhood Tourette syndrome patients and 11 (28\%) of 39 childhood chronic tic disorder patients had bipolar disorder. The difference in the prevalence of bipolar disorder was statistically significant. ${ }^{16)}$

A study of 90 adult Tourette syndrome patients undergoing regular treatments found that 30 also suffered from bipolar disorder. Bipolar disorder was most common in Tourette syndrome patients with mild tics and was associated with a wide variety of other psychopathologies. ${ }^{17}$

In a study of 156 adolescent Tourette syndrome patients, ${ }^{18)} 19(12 \%)$ patients were hospitalized, and the significant predictor of hospitalization was bipolar disorder. There is evidence to suggest that the medications used to treat Tourette syndrome may induce bipolar disorder. Patients with distinct clinical risk factors and a positive family history of ADHD and Tourette syndrome who were given guanfacine who developed hypomanic or manic symptoms. ${ }^{19)}$ Stimulants are often used to treat ADHD, even in the context of Tourette syndrome. These routinely used stimulants may precipitate bipolar disorder in vulnerable individuals and contribute to the association between Tourette syndrome and bipolar disorder. Other comorbidities encountered in Tourette syndrome patients may be important to the association between Tourette syndrome and bipolar disorder. ADHD is common in patients with Tourette syndrome and is known to be associated with bipolar disorder. ${ }^{20)}$

The systematic evaluation of bipolar disorder among childhood Tourette syndrome patients may face substantial obstacles. The combined results of 16 studies investigating mood variations in 5,409 Tourette syndrome patients found that $13-76 \%$ had a depressive disorder. ${ }^{3)}$ However, it is difficult to identify these symptoms in childhood. Childhood Tourette syndrome patients are more likely to have a depressive disorder than adolescent patients, and the symptoms and indications that define a 
hypomanic or depressive episode vary with age. Therefore, distinguishing childhood depression from other disorders is rather challenging. ${ }^{21)}$ However, $50-66 \%$ of adults report symptom onset before the age of 18 years, with $28 \%$ reporting them before 13 years of age. ${ }^{22)}$ Of children with major depressive disorder, 20-49\% experience a full manic episode by adulthood. ${ }^{23)}$ A positive family history of bipolar disorder increases the risk of mania in a depressed child; however, the exact risk in these children is not known. Proposed clinical clues for the first episode of bipolar depression include acute onset, psychosis, prominent irritability, labile mood, and poor or brief hypomanic response to antidepressants. Furthermore, features of atypical depression, including hypersomnia, hyperphagia, and other neurovegetative symptoms, may indicate a risk of future manic episodes. ${ }^{24)}$ Furthermore, for manic symptoms to be diagnosed in adolescents, euphoria, grandiosity, decreased need for sleep, or increased goal-directed activities need to be identified. ${ }^{25)}$

In the present case, the patient was admitted to a hospital on two occasions because he was uncontrollable and evidenced unstable mood, frequent excitation, and loud shouting vocal tics. Initially the patient was diagnosed with Tourette syndrome and ADHD and experienced symptoms of anxiety, frustration, depression, irritation, restlessness, instability, sleep disturbances, and coprolalia. The patient was also diagnosed with a depressive episode and was treated immediately with anti-tic and antidepressant medications, which led to symptom improvement. However, his condition was unstable, and he experienced repeated relapses resulting in treatment modifications. Prior to the patient's most recent hospitalization, he experienced a manic episode involving excitation and hyperactivity that persisted for more than one week, rendering hospitalization inevitable. Therefore, it is essential for clinicians to discriminate the depressive episodes of bipolar disorder from those of other depressive disorders in Tourette syndrome patients with accompanying anxiety and irritability before administering antidepressants. Because Tourette syndrome and bipolar disorder are closely related and often coexist, simultaneous diagnosis and treatment of these two disorders should be approached with caution.

Recently, there have been concerns about the diagnosis and treatment of bipolar disorder and early depressive episodes. Reported diagnostic procedures include measurement of amygdala and limbic system activity, with lower values indicative of a disease state, as well as identification of a short serotonin transporter gene allele. However, as there are no biological markers that can predict bipolar disorder, the diagnosis of bipolar disorder in a patient with no previous manic symptoms requires meticulous clinical examination including a thorough family history. ${ }^{26)}$

Psychosocial factors, such as stressful life events and social rhythm disruptions induced by tic symptoms are also related to bipolar disorder. ${ }^{20)}$ Therefore, while stress from tic symptoms can provoke excitation, impulsiveness, and anxiety, these conditions are indistinguishable from manic symptoms induced by Tourette syndrome itself, causing difficulty in the evaluation of bipolar disorder in a Tourette syndrome patient. The patient in the present case had tic symptoms accompanied by ADHD and suffered from anxiety, depression, excitation, and insomnia induced by stress from conflicts with his parents, resulting in his leaving school. These symptoms did improve with treatment including antidepressant and antipsychotic agents such as imipramine and sertraline. However, when the patient's medication were discontinued, he developed symptoms of euphoria, grandiosity, decreased need for sleep, and difficulty completing goal-directed activities, as well as an exacerbation of tic symptoms, especially shouting. The patient was limited in his social activities due to these symptoms.

The etiology of bipolar disorder in Tourette syndrome patients remains obscure, but the possibility of these two disorders possessing diverse but common neurophysiological properties, such as similar noradrenaline, dopaminergic, and serotonin neurotransmitter levels, remains.

Various pharmacologic and non-pharmacologic treatments for tic disorder have been reported. ${ }^{27-29)}$ The treatment of comorbid disorders in patients with tic disorders is as important as the treatment of the tic disorder, but minimal research has been carried out regarding the treatment of manic symptoms in adolescents and even less on the treatment of manic symptoms in adolescents with Tourette syndrome. Kerbeshian et al. ${ }^{15)}$ reported that the incidence and intensity of motor and vocal tics was affected by mood and both can be exacerbated in a manic state. Therefore, if treatment is focused on either the tics or the manic symptoms alone, the patient's progress will not be favorable. Aripiprazole is a partial dopamine agonist reportedly effective in patients with Tourette syndrome and manic symptoms. ${ }^{30,31)}$ Aripiprazole has been approved for the treatment of bipolar disorder in adolescents by the United States Food and Drug Administration (FDA) and was approved for treatment of bipolar and tic disorders by the Korean FDA.

It should also be noted that other agents such as risper- 
idone, ziprasidone, pimozide, haloperidol, and clonidine have produced meaningful effects in patients with chronic tics. Therefore, positive effects may also be seen in patients with Tourette syndrome and bipolar disorder treated with these drugs. ${ }^{32)}$

This case report details the diagnosis and treatment of an adolescent patient with Tourette syndrome and bipolar disorder. Current clinical research on the safety and adverse effects of the pharmacologic treatment of adolescents with Tourette syndrome and bipolar disorder is insufficient, and further research is needed.

\section{REFERENCES}

1. Sadock BJ, Sadock VA. Synopsis of Psychiatry. 9th ed. Philadelphia:Lippincott Williams \& Wilkins;2003.

2. Lewinsohn PM, Klein DN, Seeley JR. Bipolar disorders in a community sample of older adolescents: prevalence, phenomenology, comorbidity, and course. J Am Acad Child Adolesc Psychiatry 1995;34:454-463.

3. Van Meter AR, Moreira AL, Youngstrom EA. Meta-analysis of epidemiologic studies of pediatric bipolar disorder. $J$ Clin Psychiatry 2011;72:1250-1256.

4. Robertson MM. Mood disorders and Gilles de la Tourette's syndrome: An update on prevalence, etiology, comorbidity, clinical associations, and implications. $J$ Psychosom Res 2006;61:349-358.

5. Comings BG, Comings DE. A controlled study of Tourette syndrome. V. Depression and mania. Am J Hum Genet 1987;41:804-821.

6. Kerbeshian J, Burd L. Differential responsiveness to lithium in patients with Tourette disorder. Neurosci Biobehav Rev 1988; 12:247-250.

7. Kerbeshian J, Burd L. Case study: comorbidity among Tourette's syndrome, autistic disorder, and bipolar disorder. J Am Acad Child Adolesc Psychiatry 1996;35:681-685.

8. Kerbeshian J, Burd L, Klug MG. Comorbid Tourette's disorder and bipolar disorder: an etiologic perspective. Am J Psychiatry 1995;152:1646-1651.

9. Leckman JF, Riddle MA, Hardin MT. The Yale Global Tic Severity Scale; initial testing of a clinician rated scale of tic severity. J Am Acad Child Psychiatry 1989;28:566-573.

10. Young RC, Biggs T, Ziegler VE, Meyer DA. A rating scale for mania: Reliability, validity, and sensitivity. $\mathrm{Br} J$ Psychiatry 1978;133:429-435.

11. Kerbeshian J, Severud R, Burd L, Larson L. Peek-a-boo fragile site at $16 d$ associated with Tourette syndrome, bipolar disorder, autistic disorder, and mental retardation. Am J Med Genet 2000;96:69-73.

12. Mehan MR, Freimer NB, Ophoff RA. A genome-wide survey of segmental duplications that mediate common human genetic variation of chromosomal architecture. Hum Genomics 2004:1:335-344.

13. Burd L, Kerbeshian J. Gilles de la Tourette's syndrome and bipolar disorder. Arch Neurol 1984;41:1236.

14. Kerbeshian J, Burd L. Tourette disorder and bipolar symptomatology in childhood and adolescence. Can J Psychiatry 1989;34:230-233.

15. Kerbeshian J, Burd L. Comorbid Down's syndrome, Tourette syndrome and intellectual disability: registry prevalence and developmental course. J Intellect Disabil Res 2000;44:60-67. 16. Spencer T, Biederman J, Harding M, Wilens T, Faraone S. The relationship between tic disorders and Tourette's syndrome revisited. J Am Acad Child Adolesc Psychiatry 1995;34:1133-1139.

17. Berthier ML, Kulisevsky J, Campos VM. Bipolar disorder in adult patients with Tourette's syndrome: a clinical study. Biol Psychiatry 1998;43:364-370.

18. Coffey BJ, Biederman J, Geller DA, Spencer TJ, Kim GS, Bellordre CA, et al. Distinguishing illness severity from tic severity in children and adolescents with Tourette's disorder. J Am Acad Child Adolesc Psychiatry 2000;39:556-561.

19. Horrigan JP, Barnhill LJ. Guanfacine and secondary mania in children. J Affect Disord 1999;54:309-314.

20. Robertson MM. Attention deficit hyperactivity disorder, tics and Tourette's syndrome: the relationship and treatment implications. A commentary. Eur Child Adolesc Psychiatry 2006;15:1-11.

21. Carlson GA, Glovinsky I. The concept of bipolar disorder in children: a history of the bipolar controversy. Child Adolesc Psychiatr Clin N Am 2009;18:257-271.

22. Perlis RH, Miyahara S, Marangell LB, Wisniewski SR, Ostacher M, DelBello MP, et al; STEP-BD Investigators. Long-term implications of early onset in bipolar disorder: data from the first 1000 participants in the systematic treatment enhancement program for bipolar disorder (STEP-BD). Biol Psychiatry 2004;55:875-881.

23. Geller B, Zimerman B, Williams M, Bolhofner K, Craney JL. Adult psychosocial outcome of prepubertal major depressive disorder. J Am Acad Child Adolesc Psychiatry 2001;40:673-677.

24. Akiskal HS, Benazzi F. Atypical depression: a variant of bipolar II or a bridge between unipolar and bipolar II? $J$ Affect Disord 2005;84:209-217.

25. Leibenluft E, Charney DS, Towbin KE, Bhangoo RK, Pine DS. Defining clinical phenotypes of juvenile mania. Am J Psychiatry 2003;160:430-437.

26. Chang K, Howe M, Gallelli K, Miklowitz D. Prevention of pediatric bipolar disorder: integration of neurobiological and psychosocial processes. Ann N Y Acad Sci 2006; 1094:235-247.

27. Verdellen CW, Hoogduin CA, Keijsers GP. Tic suppression in the treatment of Tourette's syndrome with exposure therapy: the rebound phenomenon reconsidered. Mov Disord 2007;22:1601-1606.

28. Cianchetti C, Fratta A, Pisano T, Minafra L. Pergolide improvement in neuroleptic-resistant Tourette cases: various mechanisms causing tics. Neurol Sci 2005;26:137-139.

29. Messiha FS, Knopp W, Vanecko S, O'Brien V, Corson SA. Haloperidol therapy in Tourette's syndrome: neurophysiological, biochemical and behavioral correlates. Life Sci I 1971:10:449-457.

30. Davies L, Stern JS, Agrawal N, Robertson MM. A case series of patients with Tourette's syndrome in the United Kingdom treated with aripiprazole. Hum Psychopharmacol 2006;21:447-453.

31. Biederman J, Mick E, Spencer T, Doyle R, Joshi G, Hammerness $\mathrm{P}$, et al. An open-label trial of aripiprazole monotherapy in children and adolescents with bipolar disorder. CNS Spectr 2007;12:683-689.

32. Weisman H, Qureshi IA, Leckman JF, Scahill L, Bloch MH. Systematic review: pharmacological treatment of tic disorders--efficacy of antipsychotic and alpha-2 adrenergic agonist agents. Neurosci Biobehav Rev 2013;37:1162-1171. 